\title{
Modelagem matemática da cinética de secagem da casca do abacaxi
}

\section{Mathematical modeling for the description of drying kinetics of pineapple bark}

\author{
Daniela Dantas de Farias Leite ${ }^{1}$, Francislaine Suelia dos Santos ${ }^{2}$, Dyego da Costa Santos ${ }^{3}$, Jemima Ferreira Lisbôa ${ }^{l}$, João \\ Paulo de Lima Ferreira ${ }^{1}$, Alexandre José de Melo Queiroz ${ }^{4}$
}

Resumo: O Brasil é o segundo maior produtor de abacaxi do mundo. A polpa é a parte do fruto que é normalmente consumida, enquanto a porção mais consistente do eixo central e a casca são consideradas resíduos do seu processamento, não sendo devidamente aproveitadas pelas indústrias. Objetivou-se com este trabalho desidratar a casca do abacaxi, na forma de placas com comprimento de $15 \mathrm{~cm}$ e largura de $8 \mathrm{~cm}$, em estufa com circulação forçada de ar nas temperaturas de 75 e $85{ }^{\circ} \mathrm{C}$, e ajustando-os a modelos matemáticos. Como critério de avaliação do ajuste dos modelos matemáticos utilizou-se o coeficiente de determinação, o desvio quadrático médio e distribuição aleatória dos resíduos. A casca do abacaxi com teor de água inicial de $87,73 \%$ (b.u) apresentou teor de água final de $10,67 \%$ (b.u) na temperatura de $75{ }^{\circ} \mathrm{C}$ e $9,05 \%$ para a de $85{ }^{\circ} \mathrm{C}$, havendo uma redução média de $88,76 \%$ (b.u) do material em estudo. Verificou-se que as amostras submetidas à desidratação na temperatura $75{ }^{\circ} \mathrm{C}$ demandaram maior intervalo de tempo para atingir o teor de água de equilíbrio em comparação com as amostras desidratadas na temperatura de $85^{\circ} \mathrm{C}$. Entre os modelos estudados, observou--se que os de Page, Aproximação da Difusão e Midilli apresentaram os maiores coeficientes de determinação $\left(\mathrm{R}^{2}>0,998\right)$ e os menores desvios quadráticos médios $(\mathrm{DQM}<$ 0,02), entretanto, o modelo de Page foi o único com distribuição aleatória dos resíduos em todas as temperaturas estudadas, resultando no melhor ajuste aos dados experimentais da secagem da casca do abacaxi.

Palavras-chave: Fruta tropical; Resíduo agroindustrial; Desidratação.

\begin{abstract}
Brazil is the second largest producer of pineapple in the world. Pulp is the part of the fruit that is normally consumed, whereas the stalk and the bark are considered waste of it's processing, not being properly utilized by the industries. The objective of this work was to dehydrate the pineapple peel in the form of slices with a length of $15 \mathrm{~cm}$ and a width of $8 \mathrm{~cm}$ in an oven with forced air circulation at temperatures of 75 and $85{ }^{\circ} \mathrm{C}$ and adjust the mathematical models of Henderson and Pabis, Page, Thompson, Diffusion Approximation, Midilli and Two Terms to the experimental data. The coefficient of determination, the mean square deviation and the random distribution of the residuals were used as the criterion for evaluating the adjustment of the mathematical models. The pineapple bark with initial water content of $87.73 \%$ b.u had a final water content of $10.67 \%$ b.u at $75{ }^{\circ} \mathrm{C}$ and $9.05 \%$ at $85{ }^{\circ} \mathrm{C}$, with an average reduction of $88,76 \%$ of the initial water content of the study material. It was found that samples submitted to dewatering at $75{ }^{\circ} \mathrm{C}$ required a longer time to reach the equilibrium water content compared to dehydrated samples at a temperature of $85{ }^{\circ} \mathrm{C}$. Among the models studied, the Page of diffusion approximation and Midilli presented the highest coefficients of determination $\left(\mathrm{R}^{2}>0.998\right)$ and the smallest mean squared deviations (DQM <0.02), however, the Page model was The only one with random distribution of the residues at all temperatures studied, resulting in the best fit to the experimental data of pineapple bark drying.
\end{abstract}

Key words: Tropical fruit; Agrindustrial waste; Dehydration.

\footnotetext{
*Autor para correspondência

Recebido para publicação em 12/07/2017; aprovado em 11/09/2017

${ }^{1}$ Doutorandos, Unidade Acadêmica de Engenharia Agrícola (UAEA) do Centro de Tecnologias e Recursos Naturais (CTRN) da Universidade Federal de Campina Grande (UFCG). Campina Grande, Paraíba; Fone: (83) 99928-3848, E-mail: danieladantasfl@gmail.com; jemimaufcg_@hotmail.com; joaop_1@hotmail.com;

${ }^{2}$ Mestranda, UAEA/CTRN/UFCG. Campina Grande - PB; E-mail: suelia_santos@ hotmail.com;

${ }^{3}$ Doutor, UAEA/UFCG, Campina Grande - PB, E-mail: dyego.csantos@gmail.com;

${ }^{4}$ Professor, UAEA/UFCG, Campina Grande - PB, E-mail: alex@ deag.ufcg.edu.br
} 


\section{INTRODUÇÃO}

A produção agroindustrial no Brasil tem se expandido consistentemente a cada ano desempenhando papel superavitário na balança comercial. Favorecido pela diversidade edafoclimática, consequência da amplitude territorial, tem-se, entre nativas e exóticas, uma grande variabilidade frutífera existente no país. Essa diversidade tem motivado a implantação de inúmeras empresas alimentícias e, consequentemente, o investimento no mercado de frutas frescas e processadas, cujas atividades geram resíduos considerados como produtos de baixo valor agregado.

De acordo com a FAO (2017), o abacaxi é uma fruta tropical pertencente à família das Bromeliaceae oriunda da América do Sul. De acordo com a divisão de estatística da Organização de Agricultura e Alimentação das Nações Unidas, a produção total mundial de abacaxi entre os anos de 1994 a 2014 aumentou em 96,41\%. Em 2014, o Brasil foi o segundo maior produtor de abacaxi do mundo com cerca de 2,6 milhões de toneladas, ficando atrás, apenas, da Costa Rica, com produção de aproximadamente 2,9 milhões de toneladas no mesmo ano (FAO, 2017).

Segundo dados do Levantamento Sistemático da Produção Agrícola do Instituto Brasileiro de Geografia e Estatística, no Brasil, os principais produtores de abacaxi são os estados da Paraíba (2,68 mil toneladas), Minas Gerais (2,35 mil toneladas) e Pará (2,16 mil toneladas) (IBGE, 2017). O aumento consistente da produção de abacaxi é atribuído ao seu crescente consumo, associado a sua qualidade sensorial, apresentando polpa suculenta e saborosa e nutricionalmente valiosa, rica em ácido ascórbico, minerais, fibras e antioxidantes (RAMALLO e MASCHERONI, 2012).

Segundo Melo (2015), no processamento de frutas (polpas, sucos, doces, geleias, compotas, sorvetes, vinho, etc.) é gerada uma grande quantidade de resíduos, constituídos por talos e, principalmente, cascas, que podem corresponder entre $30 \%$ a $40 \%$ do peso da matéria prima processada. É importante ressaltar que apenas a polpa do abacaxi, seja na forma in natura ou industrializada (doces, geleias, compotas, sorvetes, vinho, etc.), é consumida, sendo a casca considerada um resíduo e descartada sem mais aplicações.

De acordo com Carvalho (2008), a casca do abacaxi apresenta em média $4,5 \%$ de proteína, $0,5 \%$ de lipídios, $3,1 \%$ de fibra bruta e 4,8\% de cinzas. Com base em suas características nutricionais, percebe-se que a casca do abacaxi pode ser considerada como um material de elevado potencial de exploração, podendo ser utilizado, quando submetido ao tratamento adequado, como matéria-prima na elaboração de novos produtos.

O abacaxi, assim como os resíduos oriundos do seu processamento, apresenta elevado conteúdo de água, sendo necessária a adoção de técnicas para prolongar sua vida útil. Dentre as tecnologias aplicadas para a conservação de alimentos, a secagem é considerada bastante eficiente, pois, além de minimizar perdas, aumentando a vida útil dos produtos alimentícios, possibilita, pela redução de massa e volume, economia no transporte e no armazenamento convertendo-os em produtos mais estáveis, promovendo a viabilidade econômica e segurança microbiológica pela eliminação da água do material, por meio da evaporação (DIÓGENES et al., 2013).

Alguns trabalhos têm sido realizados com o propósito de estudar o processo de secagem de diversos resíduos gerados pela agroindústria. Costa et al. (2007) realizaram estudo sobre a composição nutricional de pós obtidos da secagem de resíduos de abacaxi; Azoubel et al. (2008) abordaram o estudo da cinética de secagem da casca de manga "Tommy Atkins"; Ferreira e Pena (2010) estudaram a secagem da casca do maracujá amarelo; Alexandre et al. (2013) avaliaram a cinética de secagem do resíduo de abacaxi enriquecido; André et al. (2014) pesquisaram o estudo da cinética de secagem da cascas da manga espada e Costa et al. (2016) estudaram a modelagem matemática da casca da jabuticaba durante o processo de secagem.

Dessa forma, observa-se a possibilidade de se direcionar pesquisas visando o melhor aproveitamento desses resíduos, incluindo estudos no sentido de incorporá-los à alimentação humana, reduzindo a estatística de desperdício gerado pela agroindústria. Ante o exposto, e considerando o melhor aproveitamento do subproduto constituído pela casca de abacaxi, objetivou-se neste estudo desidratar a casca residual do abacaxi e ajustar diferentes modelos matemáticos aos dados experimentais.

\section{MATERIAL E MÉTODOS}

Foram utilizados abacaxis (Ananas comosus L.) da variedade Pérola, safra 2017, adquiridos na feira livre da cidade de Campina Grande, PB. Os frutos foram levados adequadamente ao laboratório, onde foram selecionados e descartados os que apresentavam injúrias mecânicas e, em seguida, realizou-se a lavagem em água corrente e sanitização em solução de hipoclorito de sódio a 100 ppm por aproximadamente $15 \mathrm{~min}$. Procedeu-se cortes longitudinais nas amostras, separando a polpa das cascas. Posteriormente, as cascas dos abacaxis foram cortadas em tamanho padronizado $(15 \times 8 \mathrm{~cm})$, colocadas em cestas teladas, aproximadamente $30 \mathrm{~g}$, e submetidas à secagem em estufa com circulação forçada de ar nas temperaturas de 75 e $85^{\circ} \mathrm{C}$. As cinéticas de secagem foram realizadas pesando-se as cestas com as amostras em intervalos regulares de 5, 10, 20, 30 e $60 \mathrm{~min}$, até atingirem o equilíbrio dinâmico com o ar de secagem. Em seguida foi determinado o teor de água das amostras em estufa a $105{ }^{\circ} \mathrm{C}$ (IAL, 2008), sendo calculadas as razões de umidade (Eq. 1) e traçadas as curvas da razão de umidade em função do tempo de secagem.

$$
\mathrm{RX}=\frac{\mathrm{X}-\mathrm{X}_{\mathrm{e}}}{\mathrm{X}_{\mathrm{i}}-\mathrm{X}_{\mathrm{e}}}
$$

Em que: RX - razão de umidade do produto (adimensional); $\mathrm{X}$ - teor de água do produto (b.s.); $\mathrm{X}_{\mathrm{i}}$ - teor de água inicial do produto (b.s.); $\mathrm{X}_{\mathrm{e}}$ - teor de água de equilíbrio do produto (b.s.).

Os modelos matemáticos descritos na Tabela 1 foram ajustados aos dados experimentais (curvas de cinética de secagem da casca do abacaxi) através de regressão não linear, pelo método Quasi-Newton, utilizando-se o programa Statistica 7.0 
Tabela 1. Modelos matemáticos utilizados para ajustar os dados da cinética de secagem da casca do abacaxi

\begin{tabular}{clc}
$\begin{array}{c}\text { Designação do } \\
\text { modelo }\end{array}$ & \multicolumn{1}{c}{ Modelo } & Eq. \\
\hline $\begin{array}{c}\text { Henderson } \mathrm{e} \\
\text { Pabis }\end{array}$ & $\mathrm{RX}=\mathrm{a} \cdot \exp (-\mathrm{k} \cdot \mathrm{t})$ & $(2)$ \\
Page & $\mathrm{RX}=\exp \left(-\mathrm{k} \cdot \mathrm{t}^{\mathrm{n}}\right)$ & $(3)$ \\
Thompson & $\left.\mathrm{RX}=\exp \left(-\mathrm{a}-\left(a^{2}+4 \cdot \mathrm{b} \cdot \mathrm{t}\right)^{0,5}\right) / 2 \cdot \mathrm{b}\right)$ & $(4)$ \\
Aproximação da & $\mathrm{RX}=\mathrm{a} \cdot \exp (-\mathrm{k} \cdot \mathrm{t})+(1-\mathrm{a}) \cdot \exp (-\mathrm{k} \cdot \mathrm{b} \cdot \mathrm{t})$ & $(5)$ \\
Difusão & $\mathrm{RX}=\mathrm{a} \cdot \exp \left(-\mathrm{k} \cdot \mathrm{t}^{\mathrm{n}}\right)+\mathrm{b} \cdot \mathrm{t}$ & $(6)$ \\
Midilli & $\mathrm{RX}=\mathrm{a} \cdot \exp \left(-\mathrm{k}_{0} \cdot \mathrm{t}\right)+\mathrm{b} \cdot \exp \left(-\mathrm{k}_{1} \cdot \mathrm{t}\right)$ & $(7)$ \\
Dois Termos &
\end{tabular}

Em que: RX - razão de teor de água, adimensional; $a, b, k, n, q-$ parâmetros dos modelos; $\mathrm{t}$ - tempo de secagem, min.

Para seleção do melhor modelo matemático foram considerados o coeficiente de determinação $\left(\mathrm{R}^{2}\right)$, o desvio quadrado médio (DQM) (Eq. 8) e a distribuição dos resíduos dos modelos ajustados às curvas de secagem. Sendo considerado como ajuste satisfatório o modelo que apresentar os maiores valores de $\mathrm{R}^{2}$, os menores valores de DQM e distribuição aleatória dos resíduos.

$$
D Q M=\sqrt{\frac{\sum(\text { RUpred }-R U \exp )^{2}}{N}}
$$

Em que: DQM: desvio quadrático médio; $\mathrm{RU}_{\text {pred }}$ : razão de umidade predita pelo modelo; $\mathrm{RU}_{\text {exp: }}$ razão de umidade experimental; N: número de observações realizadas durante o experimento.

\section{RESULTADOS E DISCUSSÃO}

Na Figura 1, verifica-se as curvas de secagem da casca do abacaxi nas temperaturas de $75^{\circ} \mathrm{C}$ e $85^{\circ} \mathrm{C}$, representadas pela razão de umidade em função do tempo de desidratação. Observa-se a influência da temperatura nas curvas de secagem, apresentando redução consistente nos tempos de desidratação com o aumento da temperatura do ar de secagem, comportamento relatado em produtos agrícolas (ALEXANDRE et al., 2013; MARTINS et al., 2014; MELO et al., 2015; GONÇALVES et al., 2016). Esse comportamento é devido à maior taxa de remoção de água do produto devido à maior transferência de energia na forma de calor, ocasionado pelo aumento da temperatura (SOUSA et al., 2011).

Figura 1. Cinética de secagem da casca do abacaxi nas temperaturas de 75 e $85^{\circ} \mathrm{C}$



Os tempos de secagem foram de 490 e 330 min nas temperaturas de $75^{\circ} \mathrm{C}$ e $85^{\circ} \mathrm{C}$, respectivamente, apresentando teor de água inicial de $87,73 \%$ b.u e final de $10,67 \%$ b.u na temperatura de $75{ }^{\circ} \mathrm{C}$ e $9,05 \%$ para a de $85{ }^{\circ} \mathrm{C}$, havendo uma redução média $88,76 \%$ do teor de água inicial do material em estudo.

Na Tabela 2 verifica-se os parâmetros dos ajustes dos modelos matemáticos de Henderson e Pabis, Page, Thompson, Aproximação da Difusão, Midilli e Dois Termos aos dados experimentais da cinética de secagem das cascas de abacaxi e seus respectivos coeficientes de determinação $\left(\mathrm{R}^{2}\right)$ e desvios quadráticos médios (DQM).

Tabela 2. Parâmetros obtidos, coeficientes de determinação $\left(\mathrm{R}^{2}\right)$, desvios quadráticos médios (DQM) dos modelos matemáticos ajustados às curvas de cinética de secagem das cascas de abacaxi

\begin{tabular}{|c|c|c|c|c|c|c|c|c|c|}
\hline \multirow{2}{*}{ Modelo } & \multirow{2}{*}{$\begin{array}{c}\mathbf{T} \\
\left({ }^{\circ} \mathbf{C}\right)\end{array}$} & \multicolumn{6}{|c|}{ Parâmetros } & \multirow{2}{*}{$\mathbf{R}^{2}$} & \multirow{2}{*}{ DQM } \\
\hline & & $\mathbf{a}$ & b & $\mathbf{k}$ & $\mathbf{k}_{0}$ & $\mathbf{k}_{1}$ & $\mathbf{n}$ & & \\
\hline \multirow{2}{*}{ Henderson e Pabis } & 75 & 1,0470 & - & 0,0131 & - & - & - & 0,9946 & 0,0222 \\
\hline & 85 & 1,0931 & - & 0,0264 & - & - & - & 0,9849 & 0,0407 \\
\hline \multirow{2}{*}{ Page } & 75 & - & - & 0,0059 & - & - & 1,1723 & 0,9991 & 0,0096 \\
\hline & 85 & - & - & 0,0053 & - & - & 1,4066 & 0,9983 & 0,0135 \\
\hline \multirow{2}{*}{ Thompson } & 75 & 0,0027 & 0,0029 & - & - & - & - & 0,8298 & 0,1364 \\
\hline & 85 & 0,0029 & 0,0038 & - & - & - & - & 0,8034 & 0,1470 \\
\hline \multirow{2}{*}{ Aproximação da Difusão } & 75 & $-84,597$ & 0,9933 & 0,0209 & - & - & - & 0,9993 & 0,0086 \\
\hline & 85 & $-59,529$ & 0,9853 & 0,0495 & - & - & - & 0,9984 & 0,0132 \\
\hline \multirow{2}{*}{ Midilli } & 75 & 0,9887 & $-0,00001$ & 0,0053 & - & - & 1,1892 & 0,9993 & 0,0087 \\
\hline & 85 & 0,9958 & 0,00003 & 0,0049 & - & - & 1,4241 & 0,9984 & 0,0131 \\
\hline \multirow{2}{*}{ Dois Termos } & 75 & 0,5259 & 0,5211 & - & 0,0131 & 0,0131 & - & 0,9955 & 0,0222 \\
\hline & 85 & 0,5466 & 0,5466 & - & 0,0263 & 0,0264 & - & 0,9849 & 0,0407 \\
\hline
\end{tabular}

Entre os modelos estudados, observa-se que os de Page, Aproximação da Difusão e Midilli apresentaram os maiores coeficientes de determinação $\left(\mathrm{R}^{2}>0,998\right)$ e os menores desvios quadráticos médios (DQM < 0,02) em todas as temperaturas, com valores bastantes próximos.
Levando em consideração esses parâmetros, todos os modelos, exceto o de Thompson, resultaram em bons ajustes aos dados experimentais da secagem das cascas do abacaxi na faixa de temperatura estudada, especialmente os três modelos citados, podendo ser utilizados para a descrição dos dados 
experimentais. Gonçalves et al. (2016), ao desidratarem a casca e polpa da banana verde (Musa acuminata) em estufa nas temperaturas de 55,65 e $75^{\circ} \mathrm{C}$, observaram que o modelo que melhor se ajustou aos dados experimentais foi o de Page. Santos et al. (2016), estudando a secagem em camada fina do fruto da palma (Opuntia fícus indica) nas temperaturas de 50, 60 e $70{ }^{\circ} \mathrm{C}$ em estufa com circulação forçada de ar, observaram que o modelo de Aproximação da Difusão apresentou o melhor ajuste aos dados experimentais. Já Costa et al. (2015), ao ajustarem diversos modelos matemáticos ao processo de secagem dos frutos de Crambe abysinica nas temperaturas de $35,45,60,75$ e $90{ }^{\circ} \mathrm{C}$ e umidades relativas de $20,9 \% ; 8,7 \% ; 6,8 \% ; 4,8 \%$ e $2,3 \%$, observaram-se que o modelo que melhor descreveu os processos de secagem em estudo foi o de Midilli.

O parâmetro "k" dos modelos Henderson e Pabis e Aproximação da Difusão aumentaram com a elevação da temperatura de secagem. Comportamento similar foi relatado por Prates et al. (2012) na secagem de folhas de Solanum lycocarpum A. St.-Hil. (fruta-de-lobo), em que o parâmetro "k" também aumentou com a elevação da temperatura de secagem para os modelos Logarítmico, Midilli, Page, Newton e Henderson e Pabis. Corrêa et al. (2010) afirmam que o parâmetro " $\mathrm{k}$ " tende a aumentar com o aquecimento, visto que maiores temperaturas acarretam maiores taxas de secagem, chegando ao teor de água de equilíbrio em menor tempo de exposição do produto ao ar de secagem. Alexandre et al. (2013), ao estudarem a secagem do resíduo de abacaxi nas temperaturas de 40,50 e $60{ }^{\circ} \mathrm{C}$, evidenciaram que o aumento da temperatura de secagem resultou no aumento da constante " $\mathrm{k}$ " nos modelos de Page, Henderson e Pabis e Lewis.

Já para os modelos de Page e Midilli foi verificado comportamento inverso, havendo a diminuição do parâmetro "k", sendo essa constante de secagem relacionada à difusividade efetiva no processo de secagem no período decrescente, sendo a difusão líquida que controla o processo de secagem (SOUSA et al., 2011). Santos et al. (2012) relatam a diminuição do parâmetro em estudo nos modelos Midilli e Page ao avaliarem a cinética de secagem de grãos residuais de urucum nas temperaturas de 40 a $70{ }^{\circ} \mathrm{C}$.

Quanto ao parâmetro " $\mathrm{n}$ " nos modelos Page e Midilli, houve o aumento de seus valores com o aumento da temperatura de secagem. Segundo Guedes e Faria (2000), o parâmetro "n" possui um efeito de moderação do tempo e corrige os prováveis erros resultantes de se negligenciar a resistência interna para a transferência de água. Comportamento semelhante também foi verificado por Pacheco et al. (2011) na secagem de cogumelos nas temperaturas de $50,60,70$ e $80^{\circ} \mathrm{C}$ e velocidades do ar de 1,5 $\mathrm{m} / \mathrm{s}$, em que o modelo de Page também apresentou aumento nos valores do parâmetro " $n$ " com a elevação da temperatura de secagem.

O parâmetro "a" dos modelos de Henderson e Pabis, Thompson, Aproximação da Difusão, Midilli e Dois termos, aumentaram com a elevação da temperatura. Tal comportamento também verificado por Rafiee et al. (2009) durante o ajuste do modelo de Page aos dados experimentais da secagem de grãos de soja.

Em relação ao parâmetro "b" nos modelos Midilli, Dois Termos e Thompson, observa-se que houve o aumento com a elevação da temperatura de secagem. Sousa et al. (2006) também verificaram, secando farelo de mamona, o aumento no parâmetro "b" do modelo de Thompson com a elevação da temperatura de secagem. Quanto ao modelo Aproximação da Difusão foi verificado comportamento inverso, havendo a diminuição de "b", corroborando com Santos et al. (2012) que observaram a diminuição do parâmetro " $b$ " nos modelos em estudo ao secar grãos residuais de urucum nas temperaturas de 40 a $70{ }^{\circ} \mathrm{C}$.

Para a seleção do melhor modelo matemático ajustado aos dados experimentais, observou-se tendência de distribuição de resíduos (Tabela 3) de todos os modelos ajustados à cinética de secagem das cascas do abacaxi. Gonelli et al. (2011) relataram que um modelo é considerado aceitável se os valores dos resíduos apresentarem distribuição aleatória. Se apresentar distribuição tendenciosa, o modelo é considerado inadequado para representar o fenômeno em questão. A não formação de figuras definidas indica aleatoriedade dos resultados (SANTOS et al., 2012).

Verifica-se que o modelo de Page foi o único com distribuição aleatória dos resíduos em todas as temperaturas estudadas, resultando no melhor ajuste aos dados experimentais da secagem da casca do abacaxi. Resultados semelhantes foram reportados por Mendonça et al. (2015) nas curvas de secagem de sementes de andiroba, onde o modelo de Page apresentou distribuição aleatória dos resíduos para todas as condições estudadas.

Tabela 3. Tendência de distribuição de resíduos para os modelos ajustados aos dados experimentais da cinética de secagem da casca do abacaxi nas temperaturas de 75 e $85^{\circ} \mathrm{C}$

\begin{tabular}{ccc}
\hline \multirow{2}{*}{ Modelo Matemático } & \multicolumn{2}{c}{ Temperatura $\left({ }^{\circ} \mathbf{C}\right)$} \\
\cline { 2 - 3 } & $\mathbf{7 5}$ & $\mathbf{8 5}$ \\
\hline Henderson e Pabis & Tendencioso & Tendencioso \\
Page & Aleatório & Aleatório \\
Thompson & Tendencioso & Tendencioso \\
Aproximação da Difusão & Aleatório & Tendencioso \\
Midilli & Aleatório & Tendencioso \\
Dois Termos & Tendencioso & Aleatório \\
\hline
\end{tabular}

Na Figura 2, observa-se as curvas de ajuste do modelo matemático de Page aos dados experimentais da cinética de secagem das cascas do abacaxi nas temperaturas de 75 e 85 ${ }^{\circ} \mathrm{C}$. Conforme o conjunto de valores dos $\mathrm{R}^{2}$ e DQM, bem como a aleatoriedade da distribuição dos resíduos, este modelo matemático foi o melhor para estimar a secagem do material em estudo nas condições experimentais deste trabalho, em que a curva do ajuste do modelo matemático ficou próxima aos dados experimentais, indicando o seu bom ajuste.

Santos et al. (2010), ao ajustarem o modelo de Page aos dados de secagem da carambola em secador de bandejas nas temperaturas de 50,60 e $70{ }^{\circ} \mathrm{C}$, também observaram bons ajustes aos dados experimentais, com valores de $\mathrm{R}^{2} \geq$ a $0,99 \mathrm{e}$ qui-quadrado $\left(\chi^{2}\right) \leq 0,001$. Martins et al. (2014), ao desidrataram a casca de mulungu (Erythrina velutina) nas temperaturas de $40,50,60$ e $70^{\circ} \mathrm{C}$ e ajustarem a diferentes modelos matemáticos os dados experimentais, verificaram que o modelo de Page representou satisfatoriamente o processo de secagem, com valores de $\mathrm{R}^{2} \geq 0,99$ e $\mathrm{DQM} \leq 0$, 03. 
Figura 2. Curvas de cinética de secagem das cascas do abacaxi nas temperaturas de 75 e $85^{\circ} \mathrm{C}$ ajustadas pelo modelo de Page

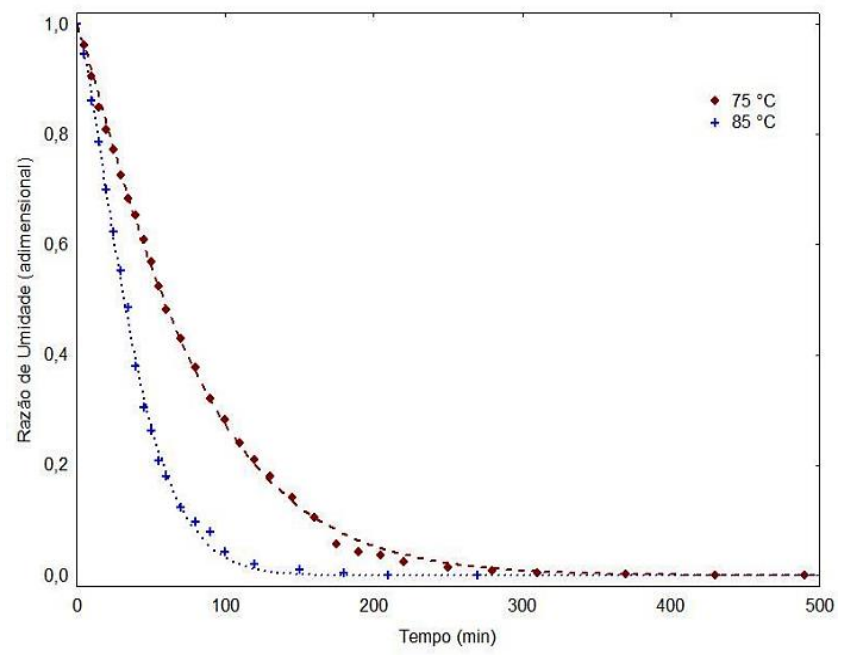

\section{CONCLUSÕES}

O aumento na temperatura de secagem promoveu redução nos tempos de desidratação.

Os modelos de Page, Aproximação da Difusão e Midilli apresentaram os maiores coeficientes de determinação $\left(\mathrm{R}^{2}>\right.$ 0,998 ) e os menores desvios quadráticos médios (DQM < 0,02), entretanto, o modelo de Page foi o único com distribuição aleatória dos resíduos em todas as temperaturas estudadas, resultando no melhor ajuste aos dados experimentais da secagem da casca do abacaxi.

\section{REFERÊNCIAS}

ALEXANDRE, H. V.; SILVA, F. L. H.; GOMES, J. P.; SILVA, O. S.; CARVALHO, J. P. D.; LIMA, E. E. Cinética de secagem do resíduo de abacaxi enriquecido. Revista Brasileira de Engenharia Agrícola e Ambiental, Campina Grande, v. 17, n. 6, p. 640-646, 2013.

ANDRÉ, A. M. M. C. N.; CASTRO, D. S.; ALMEIDA, R. D.; OLIVEIRA, V. F.; SANTOS M. I. Comparação de modelos matemáticos da cinética de secagem de cascas de Manga Espada. Revista Verde de Agroecologia e Desenvolvimento Sustentável, Pombal, v. 9, n. 3, p. 19-27, 2014.

AZOUBEL, P. M.; EVANGELISTA, E. C. D. A.; OLIVEIRA, S.B.; SILVA, Í. R. A.; ARAÚJO, A. J. B. Cinética de secagem da casca de manga "Tommy Atkins". In: Congresso Brasileiro de Engenharia Química, IV Congresso Brasileiro de Termodinâmica aplicada, 2008, Recife. Anais... Recife: UFPE: ABEQ, 2008.

CARVALHO, M. G. Barras de cereais com amêndoas de chichá, sapucaia e castanha-do-gurguéia, complementadas com casca de abacaxi. 93f. Dissertação (Mestrado em Tecnologia de Alimentos). Universidade Federal do Ceará. Fortaleza, 2008.

CORRÊA, P. C.; OLIVEIRA, G. H. H.; BOTELHO, F. M.; GONELI, A. L. D.; CARVALHO, F. M. Modelagem matemática e determinação das propriedades termodinâmicas do café (Coffea arabica L.) durante o processo de secagem. Revista Ceres, Visçosa, v. 57, n. 5, p. 595-601, 2010.

COSTA, C. F.; CORRÊA, P. C.; VANEGAS, J. D.; BAPTESTINI, F. M.; CAMPOS, R. C.; FERNANDES, L. S. Mathematical modeling and determination of thermodynamic properties of jabuticaba peel during the drying process. Revista Brasileira de Engenharia Agrícola e Ambiental, Campina Grande, v. 20, n. 6, p. 576-580, 2016.

COSTA, J. M. C.; FELIPE, E. M. DE F.; MAIA, G. A.; BRASIL, I. M.; HERNANDEZ, F. F. H. Comparação dos parâmetros físico-químicos e químicos de pós-alimentícios obtidos de resíduos de abacaxi. Revista Ciência Agronômica, v. 38 , n. 2, p.228-232, 2007.

COSTA, L. M.; RESENDE, O.; GONÇALVES, D. N.; OLIVEIRA, D. E. C. Modelagem matemática da secagem de frutos de crambe em camada delgada. Bioscience Journal, v. 31, n. 2, p. 392-403, 2015.

DIÓGENES, A. M. G.; QUEIROZ, A. J. M.; FIGUEIRÊDO, R. M. F.; SANTOS, D. C. Cinética de secagem de grãos de abóbora. Revista Caatinga, v. 26, n. 1, p. 71-80, 2013.

FERREIRA, M. D. F. P.; PENA, R. S. Estudo da secagem da casca do maracujá amarelo. Revista Brasileira de Produtos Agroindustriais, Campina Grande, v. 12, n. 1, p. 15-28, 2010. Food and Agriculture Organization of the United Nations Statistics Division - FAO. Disponível em: < http://faostat3.fao.org/browse/Q/QC/E >. Acesso em: 01.07.2017.

GONÇALVES, J. Q.; SILVA, M. A. P.; PLÁCIDO, G. R.; SILVA, M. C.; MARINS, R.; MOURA, L. C.; SOUZA, D. G. Secagem da casca e polpa da banana verde (Musa acuminata): propriedades físicas e funcionais da farinha. Global Science and Technology, v. 9, n. 3, p. 62 - 72, 2016.

GONELLI, A. L. D.; CORRÊA, P. C.; MAGALHÃES, F. E. A.; BAPTESTINI, F. M. Contração volumétrica e forma dos frutos de mamona durante a secagem. Acta Scientiarum. Agronomy, v. 33, n. 1, p. 1-8, 2011.

GUEDES, A. M. M.; FARIA, L. J. G. Determinação da constante de secagem de urucum (Bixa orellana L.) em secador convectivo de leito fixo. Revista Brasileira de Produtos Agroindustriais, Campina Grande, v. 2, n. 1, p. 7386, 2000.

IBGE. Levantamento Sistemático da Produção Agrícola. Disponível em:

ftp://ftp.ibge.gov.br/Producao_Agricola/Levantamento_Siste matico_da_Producao_Agricola_[mensal]/Fasciculo/lspa_2017 04.pdf >. Acesso em: 01.07.2017.

INSTITUTO ADOLFO LUTZ (São Paulo). Métodos físicoquímicos para análise de alimentos. São Paulo: Instituto Adolfo Lutz, 2008. 1020p.

MARTINS, J. J. A.; MARQUES, J. I.; SANTOS, D. C.; ROCHA, A. P. T. Modelagem matemática da secagem de 
cascas de mulungu. Bioscience Journal, v. 30, n. 6, p. 16521660, 2014.

MELO, J. C. S.; PEREIRA, E. D.; OLIVEIRA, K. P.; COSTA, C. H. C.; FEITOSA, R. M. Estudo da cinética de secagem da pimenta de cheiro em diferentes temperaturas. Revista Verde de Agroecologia e Desenvolvimento Sustentável, Pombal, v. 10, n. 2, p. 09 - 14, 2015.

MENDONÇA, A. P.; SAMPAIO, P. T. B.; ALMEIDA, F. A. C.; FERREIRA, R. F.; NOVAIS, J. M. Determinação das curvas de secagem das sementes de andiroba em secador solar. Revista Brasileira de Engenharia Agrícola e Ambiental, Campina Grande, v. 19, n. 4, p. 382-387, 2015.

PACHECO, A. C. W.; LUZ, G. R.; POLON, P. E.; JORGE, L. M. M.; PARAÍSO, P. R. Modeling of drying and adsorption isotherms of the fish feed. Brazilian Archives of Biology and Technology, v. 54, n. 8, p. 577-588, 2011.

PRATES, M. F. O.; REIS, R. C.; DEVILLA, I. A.; FARIA, R. Q.; LIMA JUNIOR, A. F. Cinética de secagem de folhas de Solanum lycocarpum A. St.-Hil. (fruta-de-lobo). Revista Brasileira de Plantas Medicinais, v. 14, n. 3, p. 514-521, 2012.

RAFIEE, S. H.; KEYHANI, A.; SHARIFI, M.; JAFARI, A.; MOBLI, H.; TABATABAEEFAR, A. Thin layer drying properties of soybean (Viliamz Cultivar). Journal of Agricultural Science and Technology, v. 11, n. 3, p. 289-300, 2009.

RAMALLO, L. A.; MASCHERONI, R. H. Quality evaluation of pineapple fruit during drying process. Food and Bioproducts Processing, v. 90, n. 1, p. 275-283, 2012.

SANTOS, A. E.; MARTINS, G. M. V.; CANUTO, M. F. C. S.; SEGUNDO, J. E. D. V.; ALMEIDA, R. D. Modelagem matemática para a descrição da cinética de secagem do fruto da palma (Opuntia fícus indica). Revista Verde de Agroecologia e Desenvolvimento Sustentável, Pombal, v. 11, n.1, p.01-06, 2016.

SANTOS, C. T.; BONOMO, R. F.; CHAVES, M. A.; FONTAN, R. C. I.; BONOMO, P. Cinética e modelagem da secagem de carambola (Averrhoa carambola L.) em secador de bandeja. Acta Scientiarum. Technology, v. 32, n. 3, p. 309$313,2010$.

SANTOS, D. C.; QUEIROZ, A. J. M.; FIGUEIRÊDO, R. M. F.; OLIVEIRA, E. N. A. Cinética de secagem de farinha de grãos residuais de urucum. Revista Brasileira de Engenharia Agrícola e Ambiental, Campina Grande, v. 17, n. 2, p. 223231, 2012.

SOUSA, K. A.; RESENDE, O.; CHAVES, T. H.; COSTA, L. M. Cinética de secagem do nabo forrageiro (Raphanus sativus L.). Revista Ciência Agronômica, v. 42, n. 4, p. 883-892, 2011.

SOUSA, M. B.; PEDROZA, J. P.; BELTRÃO, N. E. DE M.; SEVERINO, L. S.; DANTAS, F. P. Cinética de secagem do farelo de mamona. Revista Brasileira de Produtos Agroindustriais, Campina Grande, v. 8, n. 2, p. 135-146, 2006. 